\title{
Developing a Base Editing System to Expand the Carbon Source Utilization Spectra of Shewanella oneidensis MR-1 for Enhanced Pollutant Degradation
}

\author{
Lei Cheng ${ }^{1}$, Di Min ${ }^{1}$, Ru-Li He ${ }^{1}$, Zhou-Hua Cheng ${ }^{1}$, Dong-Feng Liu ${ }^{1}$, and Han-Qing Yu ${ }^{2}$ \\ ${ }^{1}$ University of Science and Technology of China \\ ${ }^{2}$ University of Science \& Technology of China
}

May 5, 2020

\begin{abstract}
Shewanella oneidensis MR-1, a model strain of exoelectrogenic bacteria (EEB), plays a key role in environmental bioremediation and bioelectrochemical systems because of its unique respiration capacity. However, only a narrow range of substrates can be utilized by S. oneidensis MR-1 as carbon sources, resulting in its limited applications. In this work, a rapid, highly efficient and easily manipulated base editing system pCBEso was developed by fusing a Cas9 nickase (Cas9n (D10A)) with the cytidine deaminase rAPOBEC1 in S. oneidensis MR-1. The C-to- $\mathrm{T}$ conversion of suitable $\mathrm{C}$ within the base editing window could be readily and efficiently achieved by the pCBEso system without requiring double strand break or repair templates. Moreover, double-locus simultaneous editing was successfully accomplished with an efficiency of 87.5?. With this tool, the roles of the key genes involving in N-acetyl-glucosamine (GlcNAc) or glucose metabolism in S. oneidensis MR-1 were identified. Furthermore, an engineered strain with expanded carbon source utilization spectra was constructed and exhibited a higher degradation rate for multiple organic pollutants (i.e., azo dyes and organoarsenic compounds) than the wild type when glucose or GlcNAc was used as the sole carbon source. Such a base editing system could be readily applied to other EEB. This work not only enhances the substrate utilization and pollutant degradation capacities of S. oneidensis MR-1, but also accelerates the robust construction of engineered strains for environmental bioremediation.
\end{abstract}

\section{INTRODUCTION}

Exoelectrogenic bacteria (EEB) can couple the reduction of metal oxide and electrodes with their cellular metabolism and growth, and thus play an essential role in environmental bioremediation, energy generation from wastes, and biogeochemical cycling of metals in environments ( $\mathrm{Li}, \mathrm{Yu}, \& \mathrm{He}, 2014$; Logan, 2009; Lovley, 1991; Rittmann, 2008; TerAvest \& Ajo-Franklin, 2016). Shewanella oneidensisMR-1, a model strain of EEB, can use various extracellular electron acceptors such as iron and manganese oxides, oxygen, sulfur species, radionuclides, toxic metals, and so on for cell respiration (Fredrickson et al., 2008; Nealson \& Cox, 2002). However, in contrast with its diverse electron acceptors, S. oneidensis MR-1 can use only a narrow range of substrates, such as lactate (Serres \& Riley, 2006), pyruvate (Pinchuk et al., 2009) and N-acetyl-glucosamine (GlcNAc) (Rodionov et al., 2010), as its carbon sources which significantly limits its practical applications in waste treatment and environmental bioremediation.

Carbohydrates supply carbon sources for a variety of microbes (Chaudhuri \& Lovley, 2003; Rodionov et al., 2010). Except for chitin, GlcNAc, and glycerate (Rodionov et al., 2010), S. oneidensis MR-1 can't utilize the vast majority of carbohydrates including glucose. In fact, the genomic annotations predict that S. oneidensis MR-1 has the ability to grow on glucose or maltodextrin (Rodionov et al., 2010). However, when some carbohydrates utilization pathways were reconstructed with genomic annotations, the phenotypic 
experimental results show they did not work in S. oneidensis MR-1 (Rodionov et al., 2010). Unfortunately, the genomic annotations derived from a sequence similarity analysis could not identify the inactivation of the reconstructed pathways caused by transcriptional regulations, genetic mutations, or something else (Serres \& Riley, 2006). Thus, it is necessary to rapidly identify the functions of the crucial genes involved in the carbohydrate metabolism in S. oneidensis MR-1. Then, robust engineered S. oneidensis MR-1 with an increased metabolic capacity for carbohydrates could be used to accelerate its environmental bioremediation. To achieve this goal, a rapid, highly efficient and easily tuned approach is highly desired for engineering $S$. oneidensisMR-1.

Recently, the clustered regularly interspaced short palindromic repeat (CRISPR) related systems have been widely used for targeted genome editing in numerous organisms including eukaryotes and prokaryotes (Barrangou \& Doudna, 2016; Choi \& Lee, 2016; Luo, Leenay, \& Beisel, 2016). Among these CRISPR related systems, a CRISPR/Cas9n (Cas9 D10A nickase)-mediated tool, termed "base editor", has been developed in mammalian cells and some other organisms for precise base editing (Chen et al., 2018; Komor, Kim, Packer, Zuris, \& Liu, 2016; Tong et al., 2019; Wang et al., 2018). This approach can rapidly and efficiently generate mutations without double strand break, which is required in other CRISPR/Cas9 or Cpf1-mediated genome editing systems. As a typical base editor, BE3 system developed in mammalian cells contains the engineered fusions of the Cas9n(D10A), the rat cytidine deaminase APOBEC1 (rAPOBEC1) and a uracil glycosylase inhibitor (UGI) (Komor et al., 2016). Guided by the target binding capability of Cas9n/sgRNA complex, cytidine deamination by the rAPOBEC1 occurs in the displaced single-strand DNA within the Cas9n-sgRNA-targeted DNA 'R-loop' structure (Figure 1a). The deamination of the target C in a C:G base pair results in a U:G heteroduplex, which can be converted to a T:A base pair following DNA replication (Komor et al., 2016). The C-to-T conversion of target $\mathrm{C}$ within the coding genes can rapidly result in the introduction of premature stop codons or point mutations in target genes. Thus, the base editor might be an ideal tool to quickly identify the carbohydrates metabolic pathways in S. oneidensis MR-1 and construct engineered strains with an enhanced pollutant degradation capacity.

This work aims to develop such a rapid and robust base editing system inS. oneidensis MR-1 and apply this tool to rapidly identify the functions of the genes involved in carbohydrate metabolism, and expand the metabolic capacity of $S$. oneidensisMR-1 for contaminant degradation. For this purpose, the singleplasmid-based pCBEso system was firstly constructed inS. oneidensis MR-1. Then, the effective conversions of C-to- $\mathrm{T}$ within targeted genes were confirmed and the editing efficiencies of target $\mathrm{C}$ within each $\mathrm{NC}$ motifs (N: A, T, C, G) at different positions were systematically evaluated. Furthermore, multiplex genome editing using the pCBEso system was tested and the key genes related to GlcNAc or glucose metabolism were identified. Finally, an engineered strain was constructed and its ability to degrade multiple organic pollutants was compared with the wild type when glucose or GlcNAc was used as the sole carbon source.

\section{2 | MATERIALS AND METHODS}

\subsection{Microbial strains, plasmids, and growth conditions}

All of the strains and plasmids used in this work are listed in Table S1 (Supporting Information). E. coli strains were aerobically cultured in Luria broth (LB) medium at $37{ }^{\circ} \mathrm{C}$. S. oneidensis strains were grown at $30^{\circ} \mathrm{C}$ in LB medium for preparing electrocompetent cells, or in mineral medium for degrading organic pollutants (Min et al., 2017). If necessary, appropriate chemicals were added at the following concentrations: $34 \mu \mathrm{g} / \mathrm{mL}$ chloramphenicol for E. coli Turbo, $50 \mu \mathrm{g} / \mathrm{mL}$ diaminopimelic acid and $34 \mu \mathrm{g} / \mathrm{mL}$ chloramphenicol for E. coli WM3064, and $10 \mu \mathrm{g} / \mathrm{mL}$ chloramphenicol for $S$. oneidensis MR-1.

\subsection{Construction of the pCBEso plasmid and reporter strain-MR-1/GFP-lacZ}

The pCBEso plasmid was rapidly assembled as follows: the ColE1 origin of replication and the rAPOBEC1Cas9n (D10A) expression cassette were amplified from the plasmid pnCasPA-BEC (Chen et al., 2018). The chloramphenicol-resistance marker (CmR) was copied from the pRE112 plasmid (Min et al., 2017). TherpsL promoter, controlling the transcription of the rAPOBEC1-Cas9n (D10A) expression cassette, was cloned from the genome of $S$. oneidensis MR-1. The trc promoter-driven sgRNA expression cassette, I-SceI recognition 
site, and lacpromoter-driven I-SceI Endonuclease expression cassette were synthesized by General Biosystems Co., Anhui, China. All these fragments were assembled to construct the final plasmid pCBEso via Gibson assembly method (Gibson et al., 2009). In the sgRNA expression cassette driven by the trc promoter, twoBsa I sites were introduced to facilitate the construction of targeted pCBEso plasmid via Golden Gate assembly (Engler, Kandzia, \& Marillonnet, 2008). All primers used in this work are listed in Table S2.

The GFP-lac $Z$-fused reporter was integrated into the genome of $S$. oneidensis MR-1 by homologous recombination. The plasmid pRE112-GFP-lacZ harboring two $1 \mathrm{~kb}$ homologous arms flanking the gene SO_4341 and the lacpromoter-driven GFP-lacZ expression cassette was transformed intoE. coli WM3064 and then transferred into $S$. oneidensisMR-1 by conjugation. The positive exconjugants grown on LB agar plates containing $10 \mu \mathrm{g} / \mathrm{mL}$ chloramphenicol were firstly cultured in antibiotic-free LB medium and then plated onto LB agar plates containing 10\% (wt/vol) sucrose. The chloramphenicol-sensitive and sucrose-resistant colonies were screened by PCR for the desired mutants with insertion of the GFP-lac $Z$-fused reporter.

\section{3 $\mid$ Base editing by the pCBEso system}

S. oneidensis electrocompetent cells were prepared with the following procedures: after cultivation in $30 \mathrm{~mL}$ LB medium for $16 \mathrm{~h}$, S. oneidensis cells were harvested by centrifugation (5000 g, $5 \mathrm{~min}$ ) and washed twice with $10 \mathrm{~mL}$ of sterile ice-cold $300 \mathrm{mM}$ sucrose, and finally resuspended with $2 \mathrm{~mL}$ of $300 \mathrm{mM}$ sucrose.

For the base editing in $S$. oneidensis MR-1, two single-strand DNA (ssDNA) oligos (24-nt) were designed as: 1) 5'-GTGGNNN... NNN (N20) -3'; 2) 5'-AAACNNN... NNN ( inverted repeat of N20) -3'. Then, the two ssDNA oligos were phosphorylated and annealed to form a double-strand (dsDNA) fragment with cohesive ends, which was then ligated to the $B s a$ I linearized pCBEso plasmid via Golden Gate assembly (Engler et al., 2008). The resulting pCBEso plasmid was transformed into E. coli Turbo. Then, S. oneidensiselectrocompetent cells of $100 \mu \mathrm{l}$ were mixed with the targeted pCBEso plasmid of $1 \mu \mathrm{g}$ extracted from the corresponding E. coli Turbo, and subsequently transferred into a 0.2 -cm sterile electroporation cuvette. Immediately after electroporation $\left(1.3 \mathrm{kV} \mathrm{mm}^{-1}, 100 \Omega\right.$, and $\left.25 \mu \mathrm{F}\right)$, the mixtures were supplemented with 1 $\mathrm{mL} \mathrm{LB}$ and recovered at $30^{\circ} \mathrm{C}$ for $2 \mathrm{~h}$. The cells were collected by centrifugation and then plated onto a LB agar plates containing $10 \mu \mathrm{g} / \mathrm{mL}$ chloramphenicol. Colonies grown on the plates were randomly picked and amplified by PCR. Amplified products of the targeted regions were subjected to Sanger sequencing. Similar steps were applied in the multiplex genome editing using the pCBEso system in S. oneidensis MR-1. After base editing, the targeted pCBEso plasmid was cured through the I-SceI counter-selection. The edited strains were cultured with fresh antibiotic-free LB medium containing $1 \mathrm{mM}$ isopropyl-beta-D-thiogalactopyranoside (IPTG) for $12 \mathrm{~h}$ and then plated onto the surface of LB agar plates containing $1 \mathrm{mM}$ IPTG. After incubation at $30{ }^{\circ} \mathrm{C}$ for $30 \mathrm{~h}$, the plasmid-free colonies were obtained.

To systematically evaluate the effects of the target $\mathrm{C}$ position and sequence context on the base editing efficiency, the following 7 protospacers were designed: 1) 5'- $N$ AC $\mathbf{C}_{\mathbf{1}} \mathbf{T} \mathbf{C}_{\mathbf{3}} \mathbf{G C}_{\mathbf{5}} \mathbf{C}_{\mathbf{6}} N N N \ldots N N N(12 n t) \mathbf{N G G}-3^{\prime}$; 2) 5'- $N N \mathbf{A C}_{\mathbf{2}} \mathbf{T} \mathbf{C}_{\mathbf{4}} \mathbf{G} \mathbf{C}_{\mathbf{6}} N N N \ldots N N N(12 n t) \mathbf{N G G}-3$ '; 3) 5'- $N \mathbf{C C}_{\mathbf{1}} \mathbf{A C}_{\mathbf{3}} \mathbf{T C}_{\mathbf{5}} N N N \ldots N N N(13 n t)$ NGG $-3 '$; 4) 5'- $N$ G $\mathbf{G C}_{\mathbf{1}} \mathbf{C}_{\mathbf{2}} \mathbf{A} \mathbf{C}_{\mathbf{4}} \mathbf{T} \mathbf{C}_{\mathbf{6}} N N N \ldots N N N(12 n t) \mathbf{N G G}-3 '$; 5) 5'-NN $\mathbf{G C}_{\mathbf{2}} \mathbf{C}_{\mathbf{3}} \mathbf{A} \mathbf{C}_{\mathbf{5}} N N N \ldots N N N(13 n t)$ NGG -3'; 6) 5'-N $\mathbf{T C}_{\mathbf{1}} \mathbf{G C}_{\mathbf{3}} \mathbf{C}_{\mathbf{4}} \mathbf{A} \mathbf{C}_{\mathbf{6}} N N N \ldots N N N(12 n t) \mathbf{N G G}-3$; 7 ) 5 '- $-N N \mathbf{T C}_{\mathbf{2}} \mathbf{G C}_{\mathbf{4}} \mathbf{C}_{\mathbf{5}} N N N \ldots N N N$ (13 nt) NGG -3'. All 7 suitable protospacers randomly distributed in nonessential genes were carefully screened from the genome of $S$. oneidensis MR-1 (Figure 2a).

\section{4| Phenotypic evaluations}

After overnight cultivation in $3 \mathrm{~mL} \mathrm{LB}$ medium, the edited MR-1/GFP-lac $Z$ cells were collected and washed twice by phosphate-buffered saline (PBS) buffer, and finally resuspended in $15 \mathrm{~mL}$ PBS buffer. The edited cells were measured by a Flow Cytometer (CytExpert, Beckman Coulter Inc., USA) and collected data were analyzed by CytExpert 2.0 software (CytExpert 2.0, Beckman Coulter Inc., USA). The wild-type cells were taken as a negative (GFP-) control, while the MR-1/GFP-lacZcells were taken as a positive (GFP+) control. Meanwhile, a fluorescence microscope (BX51, Olympus Inc., Japan) was used to detect the fluorescence of these cells (Cheng, Min, Liu, Li, \& Yu, 2019). In $\beta$-galactosidase activity assay, $2 \mu \mathrm{L}$ overnight culture of the positive control and edited MR-1/GFP-lac $Z$ cells was dripped onto the surface of a LB agar plate containing 
$30 \mu \mathrm{g} / \mathrm{mL}$ 5-bromo-4-chloro-3-indolyl $\beta$-D-galactopyranoside (X-gal) and incubated at $30{ }^{\circ} \mathrm{C}$ for $24 \mathrm{~h}$ for imaging.

Ampicillin susceptibility assay: overnight cultures of the wild-type and edited strains were serially diluted 10 -fold over a range of $10^{-1}$ to $10^{-6}$ with fresh LB. Then, $2 \mu \mathrm{L}$ of each diluent was dripped onto the surface of LB agar plates containing 0 or $2.5 \mu \mathrm{g} / \mathrm{mL}$ ampicillin and incubated at $30^{\circ} \mathrm{C}$ for $24 \mathrm{~h}$ for imaging.

Growth curves of the wild-type and edited strains cultured in mineral medium supplemented with GlcNAc or glucose were obtained by measuring the optical density at $600 \mathrm{~nm}\left(\mathrm{OD}_{600}\right)$ using a BioTek microplate reader (Synergy HT, BioTek Ins., USA).

\subsection{Anaerobic biodegradation oforganic pollutants}

Anaerobic reductions of organic pollutants were carried out in serum vials containing $30 \mathrm{~mL}$ mineral medium (Cheng et al., 2020; Min et al., 2017). The serum vials were sparged with $\mathrm{N}_{2}$ to maintain anaerobic conditions. Methyl orange $(\mathrm{MO}, 30 \mathrm{mg} / \mathrm{L})$, amaranth $(30 \mathrm{mg} / \mathrm{L})$, or roxarsone $(50 \mu \mathrm{M})$ was separately added into the mineral medium as the electron acceptor. The experiments for organic pollutants degradation by the wild type and the strainnagR ${ }_{\mathrm{BE}}$ in mineral medium supplemented with $20 \mathrm{mM}$ glucose were conducted as follows: the wild-type and the strain $n a g R$ BE were firstly cultured in mineral medium supplemented with $20 \mathrm{mM}$ lactate and glucose, respectively. After cultivation for $60 \mathrm{~h}$ at $30{ }^{\circ} \mathrm{C}$, the cells in mineral medium were harvested by centrifugation $(5000 \mathrm{~g}, 5 \mathrm{~min})$, washed twice by sterile mineral medium and immediately injected into the serum vials containing $30 \mathrm{~mL}$ mineral medium (with $20 \mathrm{mM}$ glucose as the sole electron donor) to reach a final $\mathrm{OD}_{600}$ of 0.3. To examine the reduction kinetics of the organic pollutants, the samples were taken from each serum vial at given time intervals. MO and amaranth were quantified and analyzed by high performance liquid chromatography (HPLC, Shimadzu Co., Japan) (Gomi et al., 2011). Roxarsone was analyzed by using a high performance liquid chromatography-hydride generation-atomic fluorescence spectrometry combined with a species analysis instrument (HPLC-HG-AFS, SAP-10, Beijing Titan Co., China), as described previously (Han et al., 2017). It was worth noting that when organic pollutants degradation abilities of the wild type and the strain $n a g R{ }_{\mathrm{BE}}$ in mineral medium supplemented with $20 \mathrm{mM}$ GlcNAc were evaluated, both strains were firstly cultured in mineral medium supplemented with $20 \mathrm{mM}$ lactate.

\section{6 $\mid$ RNA extraction and quantitative reverse transcription-PCR (qRT-PCR) analysis}

The wild-type and the strain $n a g R$ BE were cultured for $24 \mathrm{~h}$ in mineral medium with lactate as the sole carbon source. The resulting cells were collected to extract total RNA by using RNAiso plus kit (Takara Co., China). After treated with DNase I (Takara Co., China), the purified total RNA was reverse transcribed via the PrimeScript RT regent kit (Takara Co., China). qRT-PCR analysis was conducted as described previously (Liu et al., 2017). The related primers for qRT-PCR experiments are listed in Table S2.

\section{3 | RESULTS}

\subsection{Development of the base-editing system pCBEso in Shewanella oneidensis MR-1}

To perform efficient conversion of target $\mathrm{C}$ to $\mathrm{T}$ inS. oneidensis MR-1, a single-plasmid-based, CRISPR/Cas9n(D10A)-mediated base editing system pCBEso was designed and constructed (Figure 1). In the "all-in-one-plasmid" system, the cytidine deaminase rAPOBEC1 was fused to the N terminus of the Cas9n(D10A) and their expression was driven by the constitutive promoter $\left(P_{r p s L}\right)$. To apply the pCBEso plasmid for base editing of targeted genes, only a 20-bp spacer was needed to insert into the sgRNA cassette, which was controlled by the constitutive promoter $\left(P_{t a c}\right)$. A Golden Gate assembly strategy was adopted to facilitate efficient cloning of the 20-bp spacer for rapid assembly of the targeted pCBEso plasmid (Engler et al., 2008). To cure the plasmids after editing, both of I-SceI recognition site and lac promoter-driven I-SceI Endonuclease expression cassette were integrated into pCBEso plasmid. After transforming the targeted pCBEso plasmid into $S$. oneidensisMR-1, the base-editing system could readily convert the target C to $\mathrm{T}$ and enable highly efficient gene inactivation and point mutations in the targeted genes. 


\subsection{Conversion of the target $\mathrm{C}$ to $\mathrm{T}$ by the pCBEso system in $S$. oneidensis MR-1}

To find out whether the pCBEso system was an effective base editing tool, the coding region of the metalreducing/respiratory pathway was selected as a target. The reported base editors in mammalian or Streptomycetes cells have a less than 10-nucleotide editable window in the protospacer region distal to the protospacer adjacent motif (PAM) (Komor et al., 2016; Tong et al., 2019). Thus, each cytidine from Positions 1 to 10 within the protospacer in the PAM distal position was investigated (the 5 ' end of the protospacer was set to position 1, Figure S1 and Table S3). Sequencing results show that none of Cs from Positions 1, 2, 9 and 10 within the hypothetic base editing window was converted to thymidines in all 8 protospacers and the conversion of $\mathrm{C}$ to $\mathrm{T}$ was observed in the rest positions (Figure S1). The efficiency of C-to-T editing in the hypothetic base editing window was $33.3 \%$ to $100 \%$. These results suggest that the pCBEso system had a 6-nucleotide editing window from Positions 3 to 8 within the hypothetic base editing window (Figure 2a). Unexpectedly, two Cs at Positions 3 and 8 within the protospacer $m t r_{-}$Ps7 were not converted to thymidines and the target $\mathrm{C}$ was preceded by a $\mathrm{G}$ or $\mathrm{C}$. Therefore, the base editing efficiency of the pCBEso system was substantially affected by the position of target $\mathrm{C}$ and the sequence context within the base editing window.

\subsection{Characteristics ofthe pCBEso system in vivo}

In order to evaluate the effects of the target $\mathrm{C}$ position and sequence context on the base editing efficiency of the pCBEso system in vivo, a matrix was designed based on the four possible $\mathrm{NC}(\mathrm{N}: \mathrm{A}, \mathrm{T}, \mathrm{C}$, or $\mathrm{G})$ combinations of the target nucleotide with all 4 nucleotides. The target $\mathrm{C}$ of each $\mathrm{NC}$ motif occurred at all six possible positions (Figure 2a). As shown in Figure 2b, all the TC motifs within the base editing window achieved the highest conversion efficiency of C-to-T, while the editing efficiencies of the target Cs in all the GC motifs were the lowest. Overall, the editing activity of the pCBEso system on the target Cs of all four $\mathrm{NC}$ motifs follows the order $\mathrm{TC}>\mathrm{AC}>\mathrm{CC}>\mathrm{GC}$. Moreover, the target Cs at Positions 3, 4, and 5 within the 6-nucleotide editing window exhibited higher editing efficiencies than the rest.

To test the actual editing activity of the pCBEso system for the functional genes in S. oneidensis MR-1, an exogenous GFPmut3b-lac $Z$-fused reporter gene and an endogenousblaAgene were selected as targets. The protospacer in GFP gene was selected and assembled into the pCBEso plasmid (Figure 3a). Sanger sequencing results of the targeted region show that the $\mathrm{C}$ at Position 4 within the GFP spacer was successfully converted to $\mathrm{T}$ with a high editing efficiency of $4 / 4$, generating an expected STOP codon. The phenotypic changes of the edited MR-1/GFP-lac Z were further characterized by flow cytometry, chromogenic test with $\mathrm{X}$-gal, and fluorescence microscope. As shown in Figure 3b, $99.7 \%$ of edited cells collected from all four edited strains lost fluorescence, indicating that the highly efficient base editing and GFP gene inactivation occurred in the strain MR-1/GFP-lac $Z$. The X-gal reaction and fluorescence imaging results are consistent with the above observations (Figures $3 \mathrm{c}$ and $\mathrm{S} 2$ ).

BlaA, one putative $\beta$-lactamase, was reported to confer $S$. oneidensis MR-1 resistance to $\beta$-lactam (Yin et al., 2013). As shown in Figure S3a, the target $\mathrm{C}$ at Position 8 within the blaA spacer was effectively mutated to $\mathrm{T}$ with an efficiency of $3 / 3$, producing a desired STOP codon. Susceptibility assay of these strains to ampicillin was adopted to characterize the phenotypic changes of the edited strains, which further confirms the highly efficient base editing efficiency of the pCBEso system in S. oneidensis MR-1. The growth of all the three edited strains was comparable to that of the wild-type strain on LB agar plates without ampicillin addition. However, all the three edited strains, contrary to the wild-type strain, failed to grow on LB agar plates when $2.5 \mu \mathrm{g} / \mathrm{mL}$ ampicillin was added (Figure S3b). In general, the pCBEso system exhibited a highly efficient conversion of $\mathrm{C}$ to $\mathrm{T}$ within the suitable protospacers of the functional genes.

\subsection{Highly efficient double-locus base editing of $S$. oneidensis MR-1 by the pCBEso system with one single plasmid}

Compared with the conventional genetic manipulation tools, CRISPR-based genome editing methods have an unparalleled advantage in multiplex genome editing (Choi \& Lee, 2016). To achieve multiplex genome editing using the pCBEso system in $S$. oneidensis MR-1, two protospscers harboring target Cs in nagR and $d m s E$ were selected and simultaneously assembled into the pCBEso plasmid. In the resulting plasmid, 
the two sgRNAs targeting $n a g R$ and $d m s E$ were controlled by the same promoters and terminators (Figure 4a). Sanger sequencing results show that both of the target Cs at Position 3 within the nagR spacer and Position 4 within the $d m s E$ were converted to Ts with high efficiencies of $7 / 8$ and $8 / 8$, respectively (Figure 4b). Therefore, simultaneous base editing of two genes using the pCBEso system with a high efficiency in S. oneidensis MR-1 was achieved.

\subsection{Identification the key genes involved in GlcNAc or glucose metabolism by the pCBEso system in $S$. oneidensis MR-1}

As an available carbon source for $S$. oneidensis MR-1, comparative genomic analysis reveals that GlcNAc can be transported by a permease NagP into the cytoplasm and subsequently transformed to fructose 6phosphate (Fructose-6P) via an N-acetylglucosamine (NAG) catabolic pathway (Rodionov et al., 2010). Such a NAG pathway (from GlcNAc to Fructose-6P) consists of a three-step biochemical conversion (Figure 5a): 1) from GlcNAc to GlcNAc-6P (catalyzed by a GlcNAc kinase NagK); 2) from GlcNAc-6P to GlcN-6P (catalyzed by a GlcNAc-6P deacetylase NagA); and 3) from GlcN-6P to Fructose-6P (catalyzed by a GlcN$6 \mathrm{P}$ deaminase NagB) (Yang et al., 2006). Moreover, another uncharacterized membrane protein NagX was predicted to be involved in the uptake of GlcNAc (Yang et al., 2006). To examine whether NagX could be classified as a GlcNAc transporter and provide more experimental evidence on the roles of functional genes in the NAG catabolic pathway, several single or double-locus edited strains were constructed by the pCBEso system (Figures 5b and S4). The growth curves of these edited strains demonstrate thatnagK $\mathrm{BE}, n a g A{ }_{\mathrm{BE}}$, $n a g P \mathrm{BE}$ and the double-locus edited strain $n a g X / P$ BE lost the ability to grow in mineral medium with GlcNAc as the sole carbon source (Figure $5 b$ ). However, the wild type strain and nag $X{ }_{\mathrm{BE}}$ could grow on GlcNAc, indicating that NagX was not the GlcNAc transporter.

NagR, a transcriptional repressor, governs the expression of multiple genes (e.g., $\operatorname{nag} K, \operatorname{nag} B, \operatorname{nag} A, \operatorname{nag} X$ , and $\operatorname{nag} P$ ) associated with the GlcNAc metabolism in S. oneidensis MR-1 (Rodionov et al., 2010; Yang et al., 2006). Interestingly, the deletion of nagRenabled S. oneidensis MR-1 to catabolize glucose. This might be attributed to the overexpression of $\mathrm{NagP}$ and $\mathrm{NagK}$ involving in the transport and phosphorylation of glucose in MR-1, respectively (Chubiz \& Marx, 2017). In order to confirm this hypothesis, the strainnagR BEwas constructed by using the pCBEso system (Figue S5). Expectably, the expression levels ofnagK , $n a g B, \operatorname{nag} A, \operatorname{nag} X$, and $\operatorname{nag} P$ innagR BEincreased substantially than those in the wild type strain when cells were grown in mineral medium supplied with $20 \mathrm{mM}$ lactate (Figure 5c). The growth curves show that all the edited $n a g R$ BEstrains could grow on glucose, while the wild-type strain couldn't (Figure S5b). Meanwhile, two double and one triple-locus edited strains $(n a g R / P \mathrm{BE}, n a g R / X \mathrm{BE}$ and $n a g R / X / P \mathrm{BE})$ based on the strainnagR $\mathrm{BE}$ were also constructed using the pCBEso system to confirm whether NagP or NagX could transport glucose inS. oneidensis MR-1. The results suggest that NagP, rather than NagX, was the major glucose transporter in the strain nagR ${ }_{\mathrm{BE}}$ (Figure $5 \mathrm{~d}$ ). Interestingly, the deletion of nagK slightly inhibit the growth of the strainnagR $\mathrm{BE}$ on glucose, demonstrating that other unknown kinases played key roles in glucose metabolism of the strainnag $R_{\mathrm{BE}}$.

\subsection{Degradation of organic pollutants by the edited strain $n a g R_{\mathrm{BE}}$}

Firstly, several organic pollutants were selected to evaluate the degradation capacity of the strain nagR $R_{B E}$ using glucose as the sole carbon source. MO and amaranth, two typical azo dyes, could be anaerobically reduced by $S$. oneidensis MR-1 when lactate was used as the sole carbon source (Cai et al., 2012; Hong et al., 2007). HPLC analysis shows that either MO or amaranth could be degraded by the edited strain nagR $\mathrm{BE}$ when glucose was used as the sole carbon source (Figure S6). Moreover, the strainnagR BEgrown on glucose exhibited a faster MO or amaranth degradation than the wild type strain (Figure 6). The MO or amaranth degradation capacity by the wild type strain in mineral medium supplemented with glucose as the sole carbon source might be associated with the accumulation of carbon source in the inoculated culture. Meanwhile, the MO and amaranth degradation rates were also compared by calculating the first-order rate constants $(k)$ (Figure 6). The $k$ values of $\mathrm{MO}$ and amaranth degradation by the strain nagR ${ }_{\mathrm{BE}}(0.493$ $\mathrm{h}^{-1}$ and $\left.0.306 \mathrm{~h}^{-1}\right)$ were 11.7 and 7.3 times higher than that of the wild type strain $\left(0.042 \mathrm{~h}^{-1}\right.$ and 0.042 $\mathrm{h}^{-1}$ ), respectively. Previous studies demonstrated that the roxarsone, an organoarsenic compound, could be 
anaerobically degraded by $S$. oneidensis MR-1 when lactate was used as the sole carbon source (Han et al., 2017). HPLC analysis demonstrates that HAPA(V) was the main reduction product of roxarsone by the strainnagR BE when glucose was used as the sole carbon source (Figure S6). Moreover, the $k$ value of roxarsone reduction bynag $R_{\mathrm{BE}}\left(0.044 \mathrm{~h}^{-1}\right)$ was 2.4 -folds higher than that of the wild type strain $\left(0.018 \mathrm{~h}^{-1}\right)$ (Figure 6c). Overall, base editing endowed the strain nagR BE with the ability for pollutant degradation using glucose as the sole carbon source.

The increased expression levels of the genes associated with the GlcNAc metabolism in the strain $n a g R$ BE imply that such an edited strain might have a faster utilization rate for GlcNAc and thus exhibit a higher pollutant degradation capability than $S$. oneidensis MR-1 when GlcNAc was used as the sole carbon source. Expectably, the engineered strain $n a g R_{\mathrm{BE}}$ exhibited higher degradation rates for all three organic pollutants than the wild type strain (Figure 7). Meanwhile, the $k$ values of MO, amaranth, and roxarsone degradation by the strain $n a g R_{\mathrm{BE}}\left(0.711 \mathrm{~h}^{-1}, 0.469 \mathrm{~h}^{-1}\right.$, and $\left.0.089 \mathrm{~h}^{-1}\right)$ were $27.3-, 12.7$-, and 6.4 -folds higher than that of the wild type strain $\left(0.026 \mathrm{~h}^{-1}, 0.037 \mathrm{~h}^{-1}\right.$, and $\left.0.014 \mathrm{~h}^{-1}\right)$, respectively. Taken together, the base editing of $n a g R$ in $S$. oneidensis MR-1 not only expanded its carbon source utilization spectra, but also accelerated its degradation efficiencies for organic pollutants when GlcNAc was used as the sole carbon source.

\section{4 | DISCUSSION}

CRISPR/Cas9-mediated genome editing systems have been widely used in microbes (Choi \& Lee, 2016; Hong, Zhang, Cui, Wang, \& Wang, 2018; Jakociunas, Jensen, \& Keasling, 2016; Wang, Dong, Wang, Tao, \& Wang, 2017). Basically, these systems introduce a double-strand DNA break at a target position and precise genome editing is achieved by homologous recombination with a donor DNA template in the selfrepairing process of double-strand DNA break (Barrangou \& Doudna, 2016; Choi \& Lee, 2016). However, the efficiency of homologous recombination for Cas9-mediated double-strand DNA break repair in most of microbes is limited, which may cause substantial cell death. Since cytidine deamination restrictedly occurs in the displaced single-strand DNA, the pCBEso system developed in S. oneidensis MR-1 can rapidly mediate the highly efficient conversion of target $\mathrm{C}$ to $\mathrm{T}$ without requiring double-strand DNA break or a donor DNA template. In the "all-in-one-plasmid" system, a Golden Gate assembly strategy is adopted to facilitate efficient cloning of the 20-bp spacer for rapid assembly of the targeted base editing plasmid. Thus, the engineered strain can be successfully constructed by the pCBEso system within four days. In addition, multiplex genome editing of two or more loci in S. oneidensis MR-1 can be accomplished by the pCBEso system. All these features enable the pCBEso systemas a rapid, highly efficient and readily tuned approach for the identification of gene functions inS. oneidensis MR-1 and the robust construction of engineered strains.

Efforts have been made to broaden the spectra of carbohydrates as available carbon sources of $S$. oneidensis MR-1. For example, the expose to glucose enabled few $S$. oneidensis MR-1 cells to use glucose as the sole carbon source through an unknown mutation (Howard, Hamdan, Lizewski, \& Ringeisen, 2012). A similar evolution strategy was used to construct a xylose-utilizing strain by activating the silent xylose-related pathway (Sekar, Shin, \& DiChristina, 2016). An engineered Escherichia coli capable of utilizing xylose was introduced as a fermenter to synthesize lactate, which could be used by S. oneidensis MR-1 as carbon source (Yang et al., 2015). Recently, the synthetic biology strategies were adopted to engineer S. oneidensis MR-1 to respectively utilize glycerol, glucose, or xylose as the sole carbon source via heterologous expressions of modules correlated to carbohydrate metabolism (Choi et al., 2014; Flynn, Ross, Hunt, Bond, \& Gralnick, 2010; Li et al., 2017). Although these approaches are feasible, they either suffer from a low operation efficiency, or are labor intensive and time consuming. In our work, to fully exploit its intrinsic and latent carbohydrate metabolic capacity in S. oneidensis MR-1, the functions of the key genes involved in GlcNAc or glucose metabolism were quickly identified and validated by the pCBSso system. Moreover, an engineered S. oneidensis MR-1 capable of utilizing glucose was rapidly constructed, which exhibited a higher capability of biodegrading multiple organic pollutants than the wild type strain using glucose or GlcNAc as the sole carbon source. 
Despite of the above advantages, one drawback of this editing tool is the target coverage. In few non-essential genes of $S$. oneidensisMR-1, there are no editable Cs by the pCBEso system. To expand the target scope, several C-to-T base editors using Cas9 variants with different PAMs (such as VQR-BE3 and EQR-BE3) can be introduced inS. oneidensis MR-1 (Kim et al., 2017). Another possible solution to this problem is that Cas9n (D10A) with NGG in pCBEso system can be replaced by other CRISPR effectors with diverse PAMs like Cpf1 with BTTV, CasX with TTCN, and so on (Burstein et al., 2017; Yang, Gao, Rajashankar, \& Patel, 2016; Zetsche et al., 2015). Editing preference of the pCBEso system (poor conversion of target C within the GC motifs) is another shortcoming. Evolved cytosine base editors like evoAPOBEC1-BE4max and evoFERNY with highly efficient editing of target $\mathrm{C}$ within the GC context has been developed to overcome the editing preference of conventional cytosine base editors (Thuronyi et al., 2019). Recently, to write new genetic information at targeted sites in cells, a revolutionary genome editing tool (prime editing), targeting by a specific editing guide RNA, was developed by fusing a Cas9 variant with the engineered reverse transcriptase (Anzalone et al., 2019). Overall, these approaches would further expand the scopes and capabilities of base editing inS. oneidensis MR-1.

\section{5 | CONCLUSIONS}

In this work, we develop a rapid, highly efficient and readily tuned base editing system-pCBEso inS. oneidensis MR-1. Unlike other genome editing tools, the pCBEso system can achieve efficient genome editing without requiring a double-strand DNA break or repair templates. Such an effective editing system is applied to identify the key genes in GlcNAc or glucose utilization inS. oneidensis MR-1. Moreover, we rapidly construct an engineered strain for more efficient biodegradation of organic pollutants than the wild type when glucose or GlcNAc was used as the sole carbon source. In addition to the gene inactivation by introducing a premature stop codon, the pCBEso system has a great potential in repairing the undesired point mutation, engineering proteins by replacing key amino acid residues in vivo, and enhancing electron transfer pathways by multiplex genome editing in S. oneidensis MR-1 and other EEB. Such an efficient base editing system will promote the application of EEB in environmental remediation.

\section{Notes}

The authors declare no competing financial interest.

\section{ACKNOWLEDGEMENTS}

The authors wish to thank the National Key Research and Development Program of China (2018YFA0901301 and 2018YFC0406303), the National Natural Science Foundation of China (21590812, 21607146, 51878638 and 51821006), and the International Partnership Program of Chinese Academy of Sciences (GJHZ1845) for supporting this work. The authors also thank Prof. Quan-Jiang Ji from Shanghai Technological University, China, for the kind donation of the plasmid pnCasPA-BEC.

\section{Supporting Information}

Additional supporting information may be found in the online version of this article at the publisher's web-site.

\section{REFERENCES}

Anzalone, A. V., Randolph, P. B., Davis, J. R., Sousa, A. A., Koblan, L. W., Levy, J. M., Liu, D. R. (2019). Search-and-replace genome editing without double-strand breaks or donor DNA. Nature ,576 (7785), 149157. doi:10.1038/s41586-019-1711-4

Barrangou, R., \& Doudna, J. A. (2016). Applications of CRISPR technologies in research and beyond. Nature Biotechnology ,34 (9), 933-941. doi:10.1038/nbt.3659

Burstein, D., Harrington, L. B., Strutt, S. C., Probst, A. J., Anantharaman, K., Thomas, B. C., Banfield, J. F. (2017). New CRISPR-Cas systems from uncultivated microbes. Nature, 542 (7640), 237-241. doi:10.1038/nature21059 
Cai, P. J., Xiao, X., He, Y. R., Li, W. W., Chu, J., Wu, C., Yu, H. Q. (2012). Anaerobic biodecolorization mechanism of methyl orange byShewanella oneidensis MR-1. Applied Microbiology and Biotechnology , 93 (4), 1769-1776. doi:10.1007/s00253-011-3508-8

Chaudhuri, S. K., \& Lovley, D. R. (2003). Electricity generation by direct oxidation of glucose in mediatorless microbial fuel cells. Nature Biotechnology , 21 (10), 1229-1232. doi:10.1038/nbt867

Chen, W. Z., Zhang, Y., Zhang, Y. F., Pi, Y. S., Gu, T. N., Song, L. Q., Ji, Q. J. (2018). CRISPR/Cas9-based genome editing in Pseudomonas aeruginosa and cytidine deaminase-mediated base editing inPseudomonas species. iScience , 6 , 222-231. doi:10.1016/j.isci.2018.07.024

Cheng, L., Min, D., Liu, D. F., Li, W. W., \& Yu, H. Q. (2019). Sensing and approaching toxic arsenate by Shewanella putrefaciens CN-32.Environmental Science 85 Technology , 53 (24), 14604-14611. doi:10.1021/acs.est.9b05890

Cheng, Z. H., Xiong, J. R., Min, D., Cheng, L., Liu, D. F., Li, W. W., Yu, H. Q. (2020). Promoting bidirectional extracellular electron transfer of Shewanella oneidensis MR-1 for hexavalent chromium reduction via elevating intracellular cAMP level. Biotechnology and Bioengineering . doi:10.1002/bit.27305

Choi, D., Lee, S. B., Kim, S., Min, B., Choi, I. G., \& Chang, I. S. (2014). Metabolically engineered glucose-utilizing Shewanellastrains under anaerobic conditions. Bioresource Technology ,154 , 59-66. doi:10.1016/j.biortech.2013.12.025

Choi, K. R., \& Lee, S. Y. (2016). CRISPR technologies for bacterial systems: Current achievements and future directions. Biotechnology Advances , 34 (7), 1180-1209. doi:10.1016/j.biotechadv.2016.08.002

Chubiz, L. M., \& Marx, C. J. (2017). Growth trade-offs accompany the emergence of glycolytic metabolism in Shewanella oneidensis MR-1.Journal of bacteriology , 199 (11), e00827-16. doi:10.1128/JB.00827-16

Engler, C., Kandzia, R., \& Marillonnet, S. (2008). A one pot, one step, precision cloning method with high throughput capability. PLoS One, 3 (11), e3647. doi:10.1371/journal.pone.0003647

Flynn, J. M., Ross, D. E., Hunt, K. A., Bond, D. R., \& Gralnick, J. A. (2010). Enabling unbalanced fermentations by using engineered electrode-interfaced bacteria. mBio , 1 (5), e00190-10. doi:10.1128/mBio.00190-10

Fredrickson, J. K., Romine, M. F., Beliaev, A. S., Auchtung, J. M., Driscoll, M. E., Gardner, T. S., Tiedje, J. M. (2008). Towards environmental systems biology of Shewanella . Nature Reviews Microbiology , 6 (8), 592-603. doi:10.1038/nrmicro1947

Gibson, D. G., Young, L., Chuang, R. Y., Venter, J. C., Hutchison, C. A., \& Smith, H. O. (2009). Enzymatic assembly of DNA molecules up to several hundred kilobases. Nature Methods , 6 (5), 343-345. doi:10.1038/Nmeth.1318

Gomi, N., Yoshida, S., Matsumoto, K., Okudomi, M., Konno, H., Hisabori, T., \& Sugano, Y. (2011). Degradation of the synthetic dye amaranth by the fungus Bjerkandera adusta Dec 1: inference of the degradation pathway from an analysis of decolorized products. Biodegradation ,22 (6), 1239-1245. doi:10.1007/s10532011-9478-9

Han, J. C., Zhang, F., Cheng, L., Mu, Y., Liu, D. F., Li, W. W., \& Yu, H. Q. (2017). Rapid release of arsenite from roxarsone bioreduction by exoelectrogenic bacteria. Environmental Science 6 Technology Letters , 4 (8), 350-355. doi:10.1021/acs.estlett.7b00227

Hong, W., Zhang, J., Cui, G. Z., Wang, L. X., \& Wang, Y. (2018). Multiplexed CRISPR-Cpf1-mediated genome editing in Clostridium difficile toward the understanding of pathogenesis of C. difficile infection. ACS Synthetic Biology, 7 (6), 1588-1600. doi:10.1021/acssynbio.8b00087

Hong, Y. G., Guo, J., Xu, Z. C., Mo, C. Y., Xu, M. Y., \& Sun, G. P. (2007). Reduction and partial degradation mechanisms of naphthylaminesulfonic azo dye amaranth by Shewanella decolordtionis S12. Applied Microbiology and Biotechnology ,75 (3), 647-654. doi:10.1007/s00253-007-0838-7 
Howard, E. C., Hamdan, L. J., Lizewski, S. E., \& Ringeisen, B. R. (2012). High frequency of glucose-utilizing mutants in Shewanella oneidensis MR-1. FEMS Microbiology Letters , 327 (1), 9-14. doi:10.1111/j.15746968.2011.02450.x

Jakociunas, T., Jensen, M. K., \& Keasling, J. D. (2016). CRISPR/Cas9 advances engineering of microbial cell factories. Metabolic Engineering , 34 , 44-59. doi:10.1016/j.ymben.2015.12.003

Kim, Y. B., Komor, A. C., Levy, J. M., Packer, M. S., Zhao, K. T., \& Liu, D. R. (2017). Increasing the genome-targeting scope and precision of base editing with engineered Cas9-cytidine deaminase fusions. Nature Biotechnology , 35 (4), 371-376. doi:10.1038/nbt.3803

Komor, A. C., Kim, Y. B., Packer, M. S., Zuris, J. A., \& Liu, D. R. (2016). Programmable editing of a target base in genomic DNA without double-stranded DNA cleavage. Nature , 533 (7603), 420-424. doi:10.1038/nature17946

Li, F., Li, Y. X., Sun, L. M., Li, X. F., Yin, C. J., An, X. J., Song, H. (2017). Engineering Shewanella oneidensis enables xylose-fed microbial fuel cell. Biotechnology for Biofuels , 10 (1), 196. doi:10.1186/s13068017-0881-2

Li, W. W., Yu, H. Q., \& He, Z. (2014). Towards sustainable wastewater treatment by using microbial fuel cells-centered technologies.Energy $\&$ Environmental Science, 7 (3), 911-924. doi:10.1039/c3ee43106a

Liu, D. F., Min, D., Cheng, L., Zhang, F., Li, D. B., Xiao, X., Yu, H. Q. (2017). Anaerobic reduction of 2,6dinitrotoluene by Shewanella oneidensis MR-1: Roles of Mtr respiratory pathway and NfnB.Biotechnology and Bioengineering , 114 (4), 761-768. doi:10.1002/bit.26212

Logan, B. E. (2009). Exoelectrogenic bacteria that power microbial fuel cells. Nature Reviews Microbiology , 7 (5), 375-381. doi:10.1038/nrmicro2113

Lovley, D. R. (1991). Dissimilatory Fe(III) and Mn(IV) reduction. Microbiological Reviews ,55 (2), 259-287.

Luo, M. L., Leenay, R. T., \& Beisel, C. L. (2016). Current and future prospects for CRISPR-based tools in bacteria. Biotechnology and Bioengineering , 113 (5), 930-943. doi:10.1002/bit.25851

Min, D., Cheng, L., Zhang, F., Huang, X. N., Li, D. B., Liu, D. F., Yu, H. Q. (2017). Enhancing extracellular electron transfer ofShewanella oneidensis MR-1 through coupling improved flavin synthesis and metal-reducing conduit for pollutant degradation.Environmental Science \& Technology, 51 (9), 5082-5089. doi:10.1021/acs.est.6b04640

Nealson, K. H., \& Cox, B. L. (2002). Microbial metal-ion reduction and Mars: extraterrestrial expectations? Current Opinion in Microbiology , 5 (3), 296-300. doi:Doi 10.1016/S1369-5274(02)00326-0

Pinchuk, G. E., Rodionov, D. A., Yang, C., Li, X. Q., Osterman, A. L., Dervyn, E., Beliaev, A. S. (2009). Genomic reconstruction of Shewanella oneidensis MR-1 metabolism reveals a previously uncharacterized machinery for lactate utilization. Proceedings of the National Academy of Sciences of the United States of America ,106 (8), 2874-2879. doi:10.1073/pnas.0806798106

Rittmann, B. E. (2008). Opportunities for renewable bioenergy using microorganisms. Biotechnology and Bioengineering , 100 (2), 203-212. doi:10.1002/bit.21875

Rodionov, D. A., Yang, C., Li, X. Q., Rodionova, I. A., Wang, Y. B., Obraztsova, A. Y., Osterman, A. L. (2010). Genomic encyclopedia of sugar utilization pathways in the Shewanella genus. BMC Genomics , 11 (1), 494. doi:10.1186/1471-2164-11-494

Sekar, R., Shin, H. D., \& DiChristina, T. J. (2016). Activation of an otherwise silent xylose metabolic pathway in Shewanella oneidensis . Applied and Environmental Microbiology ,82 (13), 3996-4005. doi:10.1128/Aem.00881-16 
Serres, M. H., \& Riley, M. (2006). Genomic analysis of carbon source metabolism of Shewanella oneidensis MR-1: Predictions versus experiments. Journal of bacteriology , 188 (13), 4601-4609. doi:10.1128/Jb.0178705

TerAvest, M. A., \& Ajo-Franklin, C. M. (2016). Transforming exoelectrogens for biotechnology using synthetic biology.Biotechnology and Bioengineering , 113 (4), 687-697. doi:10.1002/bit.25723

Thuronyi, B. W., Koblan, L. W., Levy, J. M., Yeh, W. H., Zheng, C., Newby, G. A., Liu, D. R. (2019). Continuous evolution of base editors with expanded target compatibility and improved activity. Nature Biotechnology , 37 (9), 1070-1079. doi:10.1038/s41587-019-0193-0

Tong, Y. J., Whitford, C. M., Robertsen, H. L., Blin, K., Jorgensen, T. S., Klitgaard, A. K., Lee, S. Y. (2019). Highly efficient DSB-free base editing for streptomycetes with CRISPR-BEST. Proceedings of the National Academy of Sciences of the United States of America ,116 (41), 20366-20375. doi:10.1073/pnas.1913493116

Wang, S. H., Dong, S., Wang, P. X., Tao, Y., \& Wang, Y. (2017). Genome editing in Clostridium saccharoperbutylacetonicum N1-4 with the CRISPR-Cas9 system. Applied and Environmental Microbiology,83 (10), e00233-17. doi:10.1128/AEM.00233-17

Wang, Y., Liu, Y., Liu, J., Guo, Y. M., Fan, L. W., Ni, X. M., Ma, Y. H. (2018). MACBETH: Multiplex automated Corynebacterium glutamicumbase editing method. Metabolic Engineering , 47, 200-210. doi:10.1016/j.ymben.2018.02.016

Yang, C., Rodionov, D. A., Li, X. Q., Laikova, O. N., Gelfand, M. S., Zagnitko, O. P., Osterman, A. L. (2006). Comparative genomics and experimental characterization of N-acetylglucosamine utilization pathway of Shewanella oneidensis . Journal of Biological Chemistry,281 (40), 29872-29885. doi:10.1074/jbc.M605052200

Yang, H., Gao, P., Rajashankar, K. R., \& Patel, D. J. (2016). PAM-dependent target DNA recognition and cleavage by C2c1 CRISPR-Cas endonuclease. Cell , $16^{\text {r7 }}$ (7), 1814-1428. doi:10.1016/j.cell.2016.11.053

Yang, Y., Wu, Y. C., Hu, Y. D., Cao, Y. X., Poh, C. L., Cao, B., \& Song, H. (2015). Engineering electrodeattached microbial consortia for high-performance xylose-fed microbial fuel cell. ACS Catalysis ,5 (11), 6937-6945. doi:10.1021/acscatal.5b01733

Yin, J., Sun, L., Dong, Y., Chi, X., Zhu, W., Qi, S. H., \& Gao, H. (2013). Expression of blaA underlies unexpected ampicillin-induced cell lysis of Shewanella oneidensis .PLoS One , 8 (3), e60460. doi:10.1371/journal.pone.0060460

Zetsche, B., Gootenberg, J. S., Abudayyeh, O. O., Slaymaker, I. M., Makarova, K. S., Essletzbichler, P., Zhang, F. (2015). Cpf1 is a single RNA-guided endonuclease of a Class 2 CRISPR-Cas system. Cell ,163 (3), 759-771. doi:10.1016/j.cell.2015.09.038

\section{Figure captions}

Figure 1 The CRISPR/Cas9n (D10A)-mediated base-editing system in S. oneidensis MR-1. Strategy (a) and design (b) of the pCBEso system enabling effective conversion of $\mathrm{C}$ to $\mathrm{T}$ are shown. APOBEC1-Cas9n (D10A), a fusion protein containing the cytidine deaminase rAPOBEC1 and the Cas9 nickase (D10A); CmR, a chloramphenicol-resistance marker; ColE1, a replication origin for both of E. coli and S. oneidensis ; LacI, Lac repressor; I-SceI, the counter-selectable marker for plasmid curing after base editing. (c) Workflow of the base-editing system in S. oneidensis MR-1. Two BsaI sites were introduced into the pCBSso plasmid. After transforming the targeted pCBEso plasmid into $S$. oneidensis MR-1 by electrotransformation, the resulting cells were plated on the LB agar plates containing $10 \mu \mathrm{g} / \mathrm{mL}$ chloramphenicol. Colonies on the plates were screened and verified by PCR amplification and Sanger sequencing respectively.

Figure 2 Characteristics of the pCBEso system in S. oneidensis MR-1. (a) Seven 20-nt protospacers containing each $\mathrm{NC}$ motif at different positions were selected for evaluating the editing efficiency of the target C. The base editing window of the pCBEso systemin vivo is highlighted by gray shading. (b) Positional influences of each $\mathrm{NC}$ motif on base editing efficiency in vivo. The editing efficiencies of each NC motif 
within the base editing window are presented in a histogram. PCR products of the targeted regions in eight to ten colonies were subjected to Sanger sequencing. The optimal positions of target $\mathrm{C}$ within the base editing window are shown in yellow box.

Figure 3 Cytidine editing activity of the pCBEso system targeting an exogenous GFP-lac $Z$-fused reporter in $S$. oneidensis MR-1. (a) Sanger sequencing of the targeted region within GFP-lac $Z$-fused reporter. The protospacer is shown in red wireframe, and amino acids with their codons are indicated. PAM sequence is highlighted in red bold font. The number in red indicates positively edited clones, while the number in black represents total sequenced clones randomly picked from the plates. (b) Flow cytometry analysis of base editing activity of the pCBEso system. The wild-type and MR-1/GFP-lac Z strains were used as negative (GFP-) and positive (GFP+) controls respectively. (c) Editing efficiency of the target $\mathrm{C}$ was further confirmed by chromogenic test with X-gal.

Figure 4 Multiplex genome editing using the pCBEso system in S. oneidensis MR-1. (a) Map of the pCBEso$b l a A-d m s E$ plasmid simultaneously targeting $b l a A$ and $d m s E$. (b) Sanger sequencing of two targeted regions within $b l a A$ and $d m s E$ genes. The protospacer is shown in red wireframe, and amino acids with their codons are indicated. PAM sequence is highlighted in red bold font. The number in red indicates positively edited clones, while the number in black represents total sequenced clones randomly picked from the transformation plates.

Figure 5 (a) GlcNAc and the predicted glucose utilization pathway in S. oneidensis MR-1. Growth curves of the wild-type and edited strains cultured in mineral medium using GlcNAc (b) or glucose (d) as the sole carbon source. (c) The transcription levels of genes associated with the uptake and metabolism of GlcNAc or glucose innagR $\mathrm{BE}$ relative to those in $S$. oneidensisMR-1.

Figure 6 Anaerobic reduction of methyl orange (a), amaranth (b), and roxarsone (c) using glucose as the sole carbon source by the wild-type and engineered strain $n a g R_{\mathrm{BE}}$. 4-amino-benzenesulfonic acid (4-ABA), 4-nitro-benzene sulfonate (4-NBS) was one product of methyl orange and amaranth reduction, respectively. HAPA $(\mathrm{V})$, the main product of roxarsone reduction. The first-order rate constants $(k)$ were used to evaluate the reduction rates of different organic pollutants.

Figure 7 Anaerobic reduction of methyl orange (a), amaranth (b), and roxarsone (c) using GlcNAc as the sole carbon source by the wild-type and engineered strain $n a g R_{\mathrm{BE}}$. 4-amino-benzenesulfonic acid (4-ABA), 4-nitro-benzene sulfonate (4-NBS) was one product of methyl orange and amaranth reduction, respectively. HAPA $(\mathrm{V})$, the main product of roxarsone reduction. The first-order rate constants $(k)$ were used to evaluate the reduction rates of different organic pollutants. 
(a)

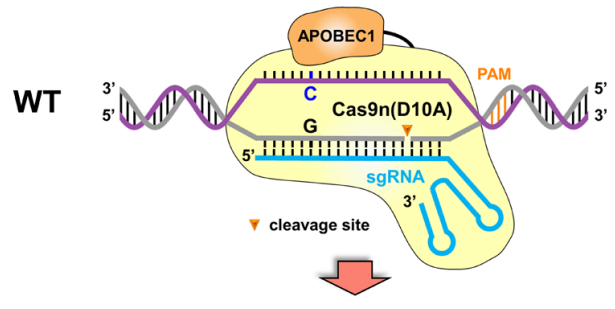

Edited ${ }_{5}^{3}, D N D$ NDN

(c)

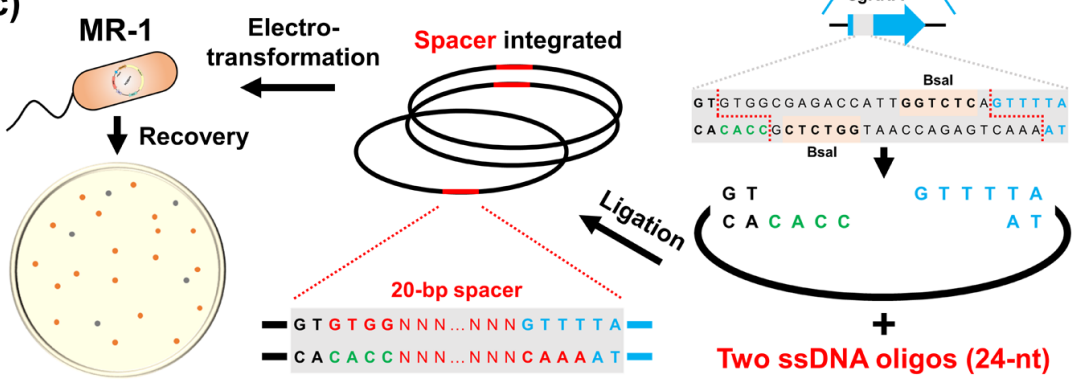

(b)

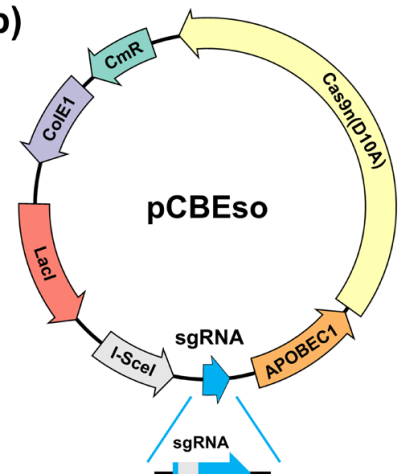

GT: TGGCGAGACCATTGGTCTCAOTTTTA CACACC:GCTCTGGTAACCAGAGTCAAA:A .

Figure 1

(a) Base editing window

\begin{tabular}{c|c|c|c|c|c|c|c|c|c|c|c|c|c|c|c|c|c|c|c|c|}
\hline 1 & 2 & 3 & 4 & 5 & 6 & 7 & 8 & 9 & 10 & 11 & 12 & 13 & 14 & 15 & 16 & 17 & 18 & 19 & 20 & PAM \\
\hline $\mathrm{A}$ & $\mathrm{A}$ & $\mathrm{C}_{3}$ & $\mathrm{~T}$ & $\mathrm{C}_{5}$ & $\mathrm{G}$ & $\mathrm{C}_{7}$ & $\mathrm{C}_{8}$ & $\mathrm{~A}$ & $\mathrm{C}$ & $\mathrm{G}$ & $\mathrm{C}$ & $\mathrm{C}$ & $\mathrm{G}$ & $\mathrm{A}$ & $\mathrm{G}$ & $\mathrm{A}$ & $\mathrm{T}$ & $\mathrm{C}$ & $\mathrm{T}$ & TGG \\
\hline $\mathrm{A}$ & $\mathrm{A}$ & $\mathrm{A}$ & $\mathrm{C}_{4}$ & $\mathrm{~T}$ & $\mathrm{C}_{6}$ & $\mathrm{G}$ & $\mathrm{C}_{8}$ & $\mathrm{C}$ & $\mathrm{C}$ & $\mathrm{A}$ & $\mathrm{G}$ & $\mathrm{C}$ & $\mathrm{C}$ & $\mathrm{C}$ & $\mathrm{A}$ & $\mathrm{T}$ & $\mathrm{G}$ & $\mathrm{C}$ & $\mathrm{G}$ & CGG \\
\hline $\mathrm{A}$ & $\mathrm{C}$ & $\mathrm{C}_{3}$ & $\mathrm{~A}$ & $\mathrm{C}_{5}$ & $\mathrm{~T}$ & $\mathrm{C}_{7}$ & $\mathrm{G}$ & $\mathrm{C}$ & $\mathrm{C}$ & $\mathrm{T}$ & $\mathrm{G}$ & $\mathrm{T}$ & $\mathrm{T}$ & $\mathrm{T}$ & $\mathrm{A}$ & $\mathrm{A}$ & $\mathrm{C}$ & $\mathrm{G}$ & $\mathrm{C}$ & CGG \\
\hline $\mathrm{C}$ & $\mathrm{G}$ & $\mathrm{C}_{3}$ & $\mathrm{C}_{4}$ & $\mathrm{~A}$ & $\mathrm{C}_{6}$ & $\mathrm{~T}$ & $\mathrm{C}_{8}$ & $\mathrm{G}$ & $\mathrm{C}$ & $\mathrm{C}$ & $\mathrm{T}$ & $\mathrm{T}$ & $\mathrm{C}$ & $\mathrm{A}$ & $\mathrm{C}$ & $\mathrm{G}$ & $\mathrm{C}$ & $\mathrm{A}$ & $\mathrm{A}$ & TGG \\
\hline $\mathrm{G}$ & $\mathrm{A}$ & $\mathrm{G}$ & $\mathrm{C}_{4}$ & $\mathrm{C}_{5}$ & $\mathrm{~A}$ & $\mathrm{C}_{7}$ & $\mathrm{~T}$ & $\mathrm{C}$ & $\mathrm{G}$ & $\mathrm{T}$ & $\mathrm{A}$ & $\mathrm{G}$ & $\mathrm{T}$ & $\mathrm{T}$ & $\mathrm{A}$ & $\mathrm{C}$ & $\mathrm{G}$ & $\mathrm{G}$ & $\mathrm{T}$ & CGG \\
\hline $\mathrm{A}$ & $\mathrm{T}$ & $\mathrm{C}_{3}$ & $\mathrm{G}$ & $\mathrm{C}_{5}$ & $\mathrm{C}_{6}$ & $\mathrm{~A}$ & $\mathrm{C}_{8}$ & $\mathrm{~T}$ & $\mathrm{C}$ & $\mathrm{A}$ & $\mathrm{A}$ & $\mathrm{T}$ & $\mathrm{G}$ & $\mathrm{T}$ & $\mathrm{G}$ & $\mathrm{G}$ & $\mathrm{A}$ & $\mathrm{A}$ & $\mathrm{C}$ & TGG \\
\hline $\mathrm{G}$ & $\mathrm{A}$ & $\mathrm{T}$ & $\mathrm{C}_{4}$ & $\mathrm{G}$ & $\mathrm{C}_{6}$ & $\mathrm{C}_{7}$ & $\mathrm{~A}$ & $\mathrm{C}$ & $\mathrm{T}$ & $\mathrm{T}$ & $\mathrm{T}$ & $\mathrm{A}$ & $\mathrm{G}$ & $\mathrm{T}$ & $\mathrm{G}$ & $\mathrm{C}$ & $\mathrm{G}$ & $\mathrm{C}$ & $\mathrm{A}$ & TGG \\
\hline
\end{tabular}

(b)

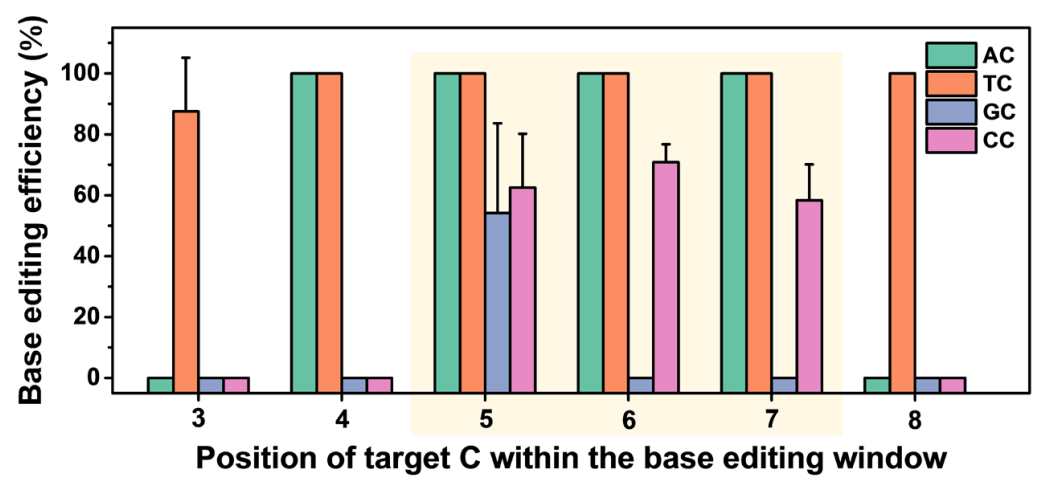

Figure 2 
(a)

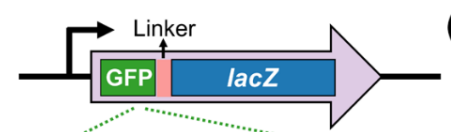

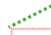

MR-1/ TATCAACAAAATACTCCGATTGGC GFP-lacZ ATAGTTGTTTTATGAGGCTAACCG \begin{tabular}{llllll}
1 & 1 & 185 & 1 & 1 \\
\hline Tyr Gln GIn Asn Thr Pro lle Gly
\end{tabular} TATCAACAAAATACTCCGATTGGC

Edited TATTAACAAAATACTCCGATTGGC $4 / 4$ STOP

(c)

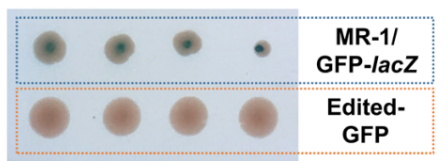

(b)
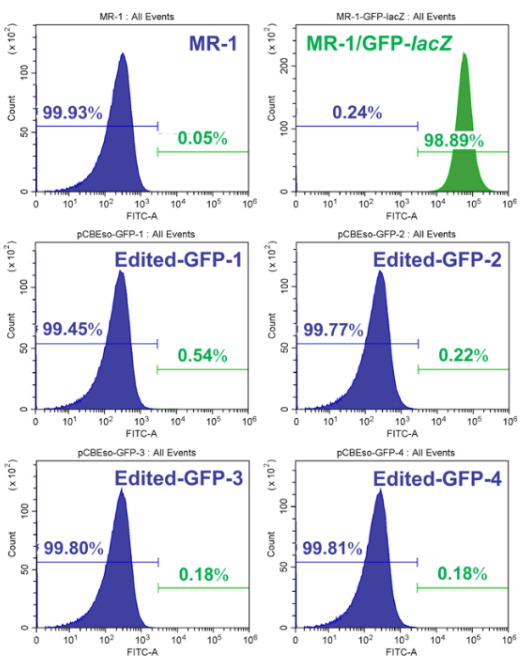

\section{Figure 3}

(a)

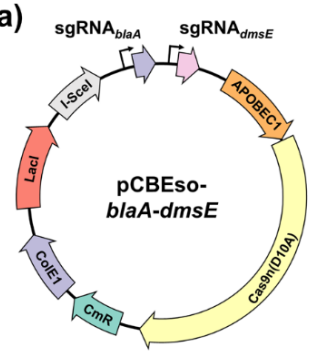

(b)

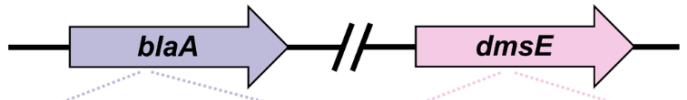

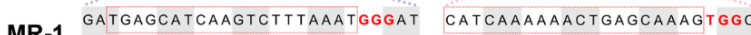

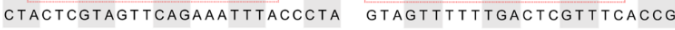

\begin{tabular}{l} 
Asp Glu His \\
\hline
\end{tabular} MR-1 GATGAGCATCAAGTCTTTAAATGGGAT CATCAAAAAACTGAGCAAAGTGGC Edited GATGAGCATTAAGTCTTTAAATGGGAT CATTAAAAAACTGAGCAAAGTGGC

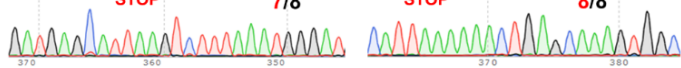

Figure 4 
(a)

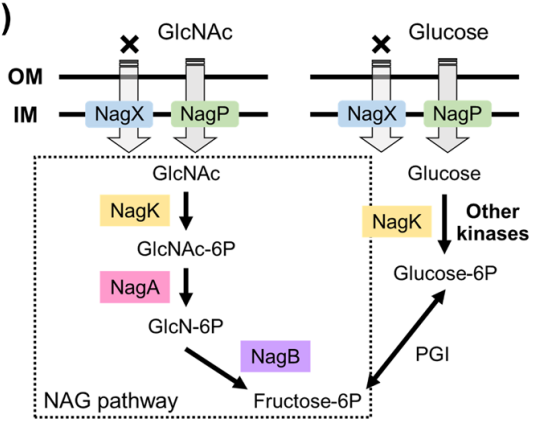

(c)

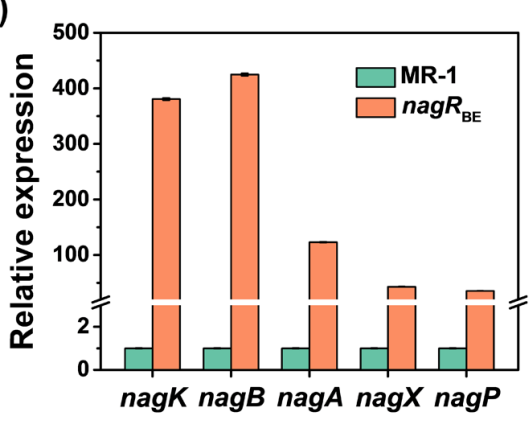

(b)

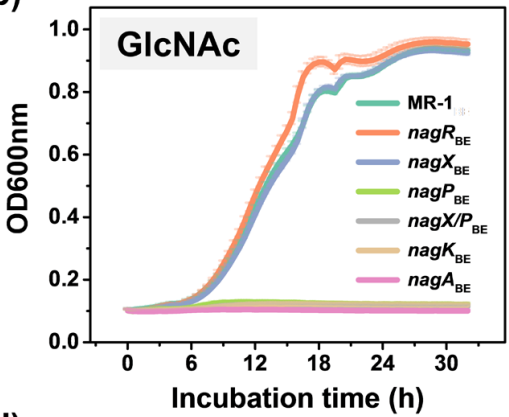

(d)

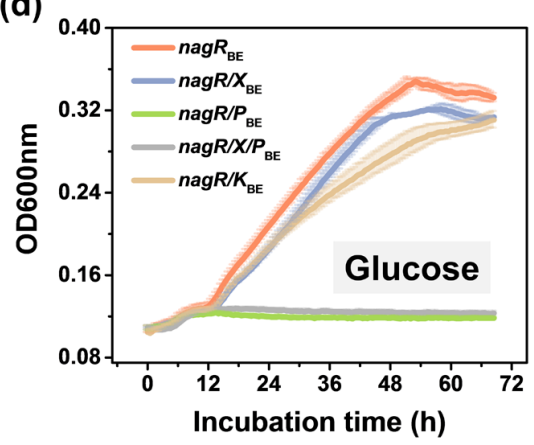

Figure 5 
(a)

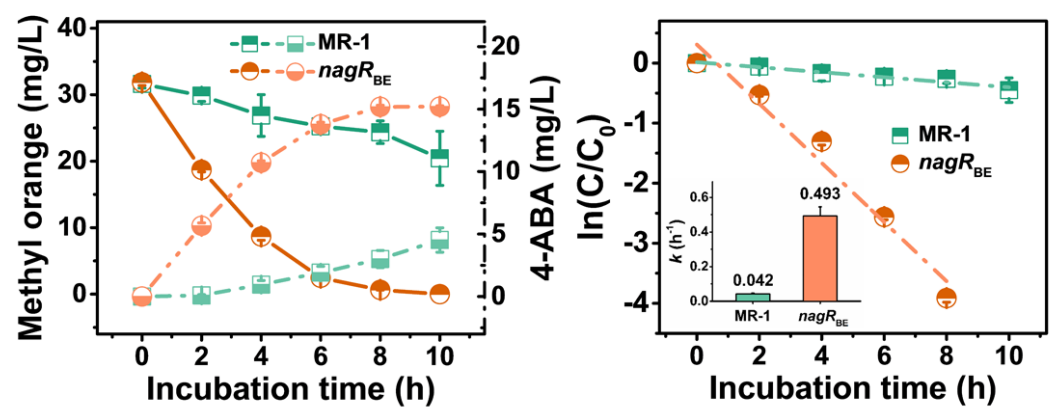

(b)

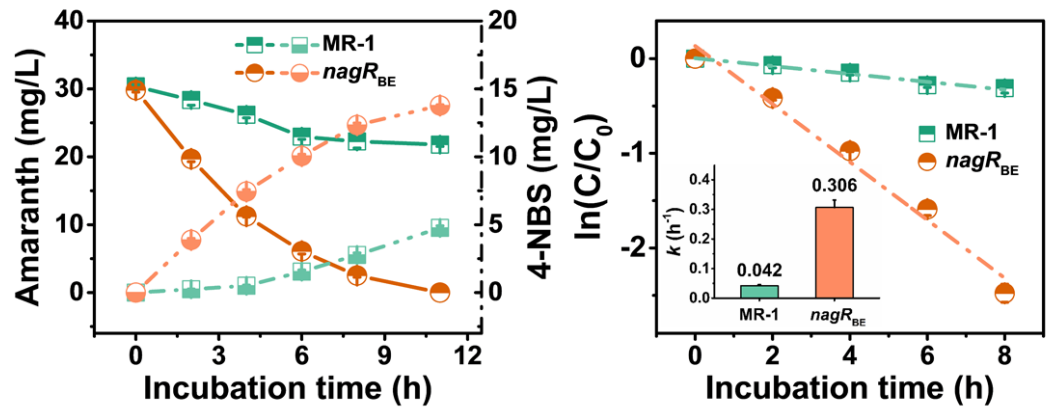

(c)

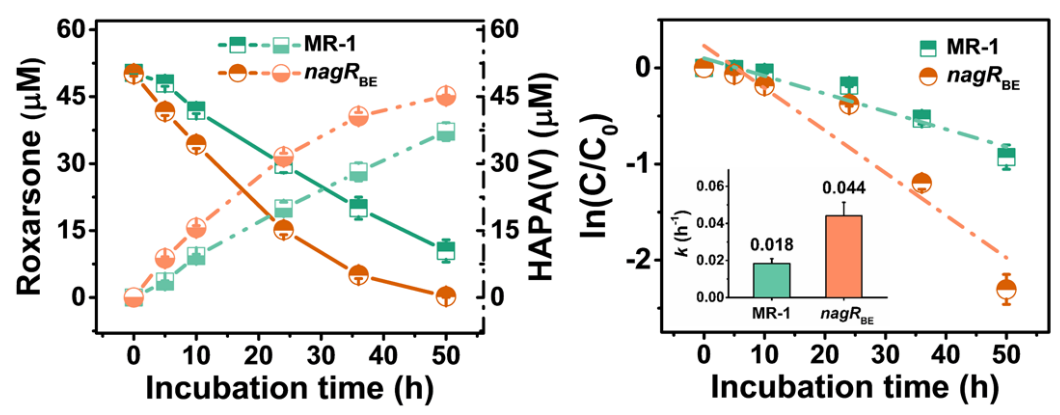

Figure 6 
(a)

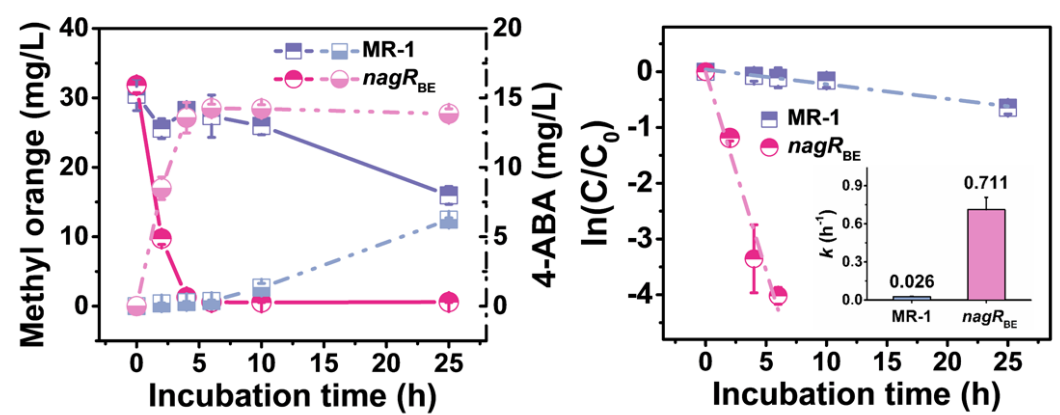

(b)

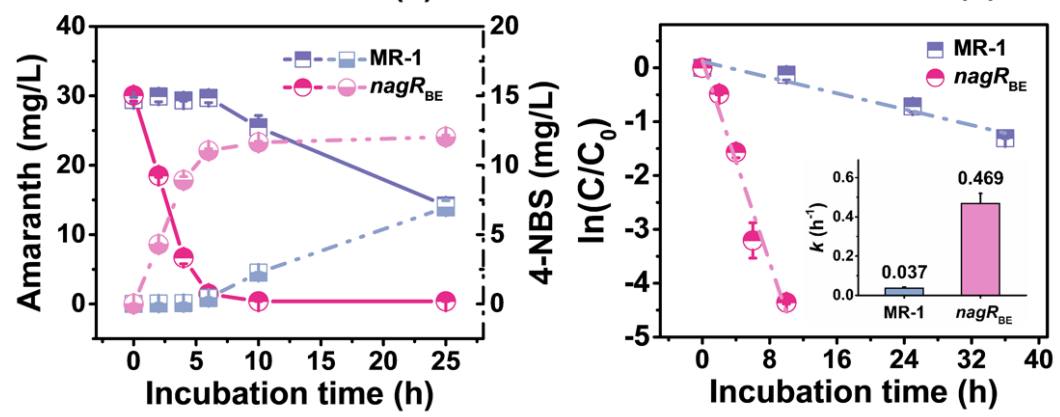

(c)

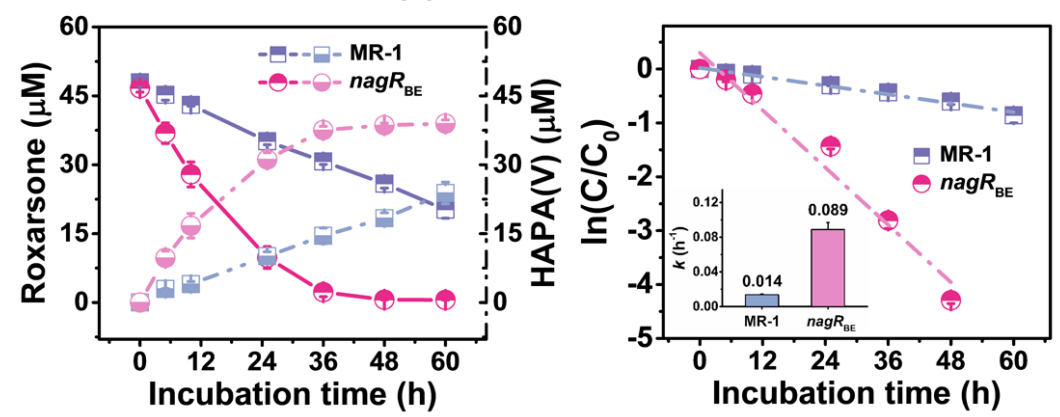

Figure 7 